\title{
Network Design Algorithm Implementation for Resilient Transportation System under Continuous Risk Perturbation with Big Data Analysis
}

\author{
Hongxiao Wang $\mathbb{D}^{1,2}{ }^{1,2}$ Qiang $\mathrm{Li}^{3}$ and Sang-Bing Tsai $\mathbb{i D}^{4}$ \\ ${ }^{1}$ College of Transportation Engineering, Chang'an University, Xi'an 710061, China \\ ${ }^{2}$ Department of Mechanical and Traffic Engineering, Ordos Institute of Technology, Ordos 017010, China \\ ${ }^{3}$ Public Security Bureau Traffic Management Detachment of Ordos, Ordos 017010, China \\ ${ }^{4}$ Regional Green Economy Development Research Center, School of Business, WUYI University, Nanping, China
}

Correspondence should be addressed to Hongxiao Wang; whxtc06@163.com

Received 27 October 2021; Revised 18 November 2021; Accepted 1 December 2021; Published 4 January 2022

Academic Editor: Xiangtao Li

Copyright (c) 2022 Hongxiao Wang et al. This is an open access article distributed under the Creative Commons Attribution License, which permits unrestricted use, distribution, and reproduction in any medium, provided the original work is properly cited.

\begin{abstract}
With the rapid economic development and urbanization process accelerating, motor vehicle ownership in large cities is increasing year by year; urban traffic congestion, parking difficulties, and other problems are becoming increasingly serious; in ordinary daily life, continuous risk of disturbance, having a flexible transportation system network is more able to alleviate daily congestion in the city, and the main thing about flexible transportation network is its algorithm. It is worth noting that congestion in many cities is generally reflected in the main roads, while many secondary roads and branch roads are underutilized, and the limited road resources in cities are not fully utilized. As an economic and effective road traffic management measure, one-way traffic can balance the spatial and temporal distribution of traffic pressure within the road network, make full use of the existing urban road network capacity, and solve the traffic congestion problem. Therefore, it is of great theoretical and practical significance to develop a reasonable and scientific one-way traffic scheme according to the characteristics of traffic operation in different regions. Based on the fixed demand model, the influence of traffic demand changes is further considered, the lower-level model is designed as an elastic demand traffic distribution model, the excess demand method is used to transform the elastic demand problem into an equivalent fixed demand problem based on the extended network, and the artificial bee colony algorithm based on risk perturbation is designed to solve the two-level planning model. The case study gives a one-way traffic organization optimization scheme that integrates three factors, namely, the average load degree overload limit of arterial roads, the detour coefficient, and the number of on-street parking spaces on feeder roads, and performs sensitivity analysis on the demand scaling factor.
\end{abstract}

\section{Introduction}

With the rapid economic development, motor vehicle ownership in large cities has increased year by year, and traffic problems have become a common problem faced by countries around the world in the process of urban development. In a megacity, for example, motor vehicle ownership reached 6.084 million in 2018, an increase of 3\% over the previous year; among them, private motor vehicle ownership reached 4.894 million, an increase of $2.9 \%$ over last year [1]. At present, traffic congestion and parking difficulties in large cities are becoming increasingly serious, mainly because the limited traffic resources cannot meet the growing traffic demand of the public and the consequent increase in travel costs, traffic accident rates are rising year by year, and many other problems not only reduce the overall operational efficiency of the urban transportation system but also restrict the rapid development of the economy and the city, which has continuous risk disturbance in terms of traffic. By the end of 2018, the total road mileage in its urban area was $6203 \mathrm{~km}$, of which $390 \mathrm{~km}$ was urban expressways, $998 \mathrm{~km}$ was urban trunk roads, $632 \mathrm{~km}$ 
was urban secondary roads [2], and $4183 \mathrm{~km}$ was branch roads and below; the total road area was 103.28 million $\mathrm{m}^{2}$. the average traffic index in the central urban area during peak hours was 5.5. The road traffic congestion in the evening peak was higher than that in the morning peak, and the annual average road traffic index in the morning peak was 5.1, while that in the evening peak was 5.1. The average annual road traffic index is 5.1 in the morning peak and 6.0 in the evening peak, and the average duration of congestion (including severe congestion and moderate congestion) is $2 \mathrm{~h} 50 \mathrm{~min}$. How to effectively improve urban traffic operations has gradually become a focus of widespread attention from the government and the community [3].

The application of big data is a very good tool for traffic management departments. The current solution for traffic congestion is to build new roads, which costs a lot. Based on big data, the method of rationally diverting vehicles and alleviating the pressure of traffic is proposed. To solve the traffic problem, the government has invested a lot of money in the improvement and expansion of roads. $35 \mathrm{~km}$ of new urban roads was added in its city in 2018, and the total mileage reached $6395 \mathrm{~km}$. To solve the traffic congestion problem, governments at all levels have formulated many measures, such as motor vehicle restrictions and increased parking fees, which have alleviated traffic congestion to some extent, but have not fundamentally solved the traffic congestion problem. Traffic congestion is still serious during holidays and morning and evening rush hours, and the urban traffic problem needs to be solved urgently. Applying multiple traffic assignment models to urban road traffic network design is beneficial to improve the accuracy of investment decisions [4]. The study of traffic network design is based on system engineering as the starting point, and modern optimization methods are used to find the best investment plan for expanding or building new roads, maximize the effect of limited funds, and optimize certain indicators of the studied traffic network. In previous studies, experts and scholars have focused on proposing different types of traffic network indicators, considering more traffic network design constraints, or proposing more effective solution algorithms, but less on the relationship between traffic network design solutions and traveler behavior. Traveler behavior has a significant impact on the outcome of the design solution, and the adoption of a traffic assignment model that can more accurately describe the traffic distribution in the studied traffic network is a key point to ensure the success of traffic network design and urban transportation planning [5].

For urban traffic problems, a lot of researches have been conducted in the following two aspects: firstly, from the perspective of the flexible transportation system, rational planning of urban space to improve the supply of transportation facilities; secondly, from the perspective of demand management, balancing transportation supply and demand while reducing transportation demand. It has been proved that the investment in traffic infrastructure cannot fundamentally solve the traffic problem, and the increase of traffic supply will induce new travel demand, which will lead to a vicious circle of "increase supply-stimulate traffic demand-increase supply again," and road reconstruction and expansion and urban development and land use are closely related and cannot be increased indefinitely.

Therefore, a two-pronged approach is needed to solve the traffic problem, focusing on both infrastructure development and the flexible use of traffic demand management measures. At the same time, it is worth noting that congestion in many cities is generally reflected in the main roads, while many secondary and feeder roads are underutilized, and the limited road resources in cities are not fully utilized [6].

One-way traffic, as an economic and effective road traffic management measure, is one of the traffic organization methods often used in traffic microcirculation design, which can balance the spatial and temporal distribution of traffic pressure within the road network and improve the service level of bottleneck sections and intersections.

Improving the design of a flexible traffic system network, making the traffic network more flexible, can solve the problem of traffic congestion to a certain extent. Scientific and reasonable organization of one-way traffic can make full use of the existing urban road network capacity and effectively alleviate urban traffic congestion. To solve the traffic congestion problem, exploring scientific and comprehensive one-way traffic organization scheme optimization process has become a hot spot for scholars at home and abroad to study how to develop a reasonable one-way traffic organization optimization scheme to achieve the expected effect of alleviating traffic congestion and other problems, which has important theoretical and practical significance [7].

\section{Related Work}

With the famous deterministic user equilibrium (UE) model proposed in the literature, the study of traffic assignment has entered a completely new phase. A mathematical model of the user balance principle was proposed in the literature, and the model was successfully solved using the Frank-Wolfe algorithm in the literature and others. The deterministic user equilibrium model assumes that the traveler is fully aware of the road conditions and makes a completely correct path choice, which is not in line with reality. There is some error between the traveler's estimate of the path impedance and the actual value. Based on this, the literature proposes a stochastic user equilibrium (SUE) model, where the SUE still satisfies the user equilibrium principle, but the traveler selects the path according to the minimum impedance he/she perceives [8]. The literature proposed an equivalent mathematical planning model for SUE, which includes other models for path selection that satisfy the translation-invariant distribution in addition to the Logit and Probit models. The literature designed the Method of Successive Averages (MSAs) to successfully solve the SUE model, which is widely used in traffic assignment studies to subdivide travelers into four types according to the finite rationality of travelers' path selection and the variability of risk sensitivity coefficients, establish the prospect values of each type of travelers, and construct a stochastic user equilibrium model. The results of the algorithm show that the setting of the 
cumulative prospect theory parameters has an important influence on the results of traffic assignment. The literature proposed a stochastic user balance model with exponential pheromone update strategy and solved the model using the successive averaging (MSA) and ant colony algorithm and finally compared the output results and algorithm sensitivity analysis. Literature [9] constructed a hierarchical Logitbased SUE model by considering the path selection and mode choice behaviors of travelers and designed an improved direction search algorithm to solve it.

The most basic one is the elastic demand-based user equilibrium model, which can be solved to obtain the roadway flow and OD demand satisfying the user equilibrium principle, and the OD demand must also satisfy the defined demand function, a multicategory, multicriteria transportation network equilibrium model considering elastic demand, and the demand function usually depends on the negative utility of all categories among ODs. An efficient algorithm for computing this model is proposed in the literature [10]. A multiuser stochastic user equilibrium model based on the elastic demand allocation criterion is constructed, an efficient path search algorithm is designed to solve it, and numerical simulations are performed by arithmetic examples. Someone constructs an elastic demand combination model with the coexistence of user balance and stochastic user balance and designs a diagonalization and MSA combination algorithm. For the elastic demand asymmetric stochastic user balance problem, a Variational Inequality (VI) model is constructed through Probit-based stochastic network loading, a Monte Carlo simulation method is proposed to solve the model, and a path-based Gradient Projection (GP) algorithm is used to solve the elastic demand traffic assignment problem of the large-scale road network, the elastic demand user equilibrium assignment problem is studied under the road section capacity constraint, the excess demand model is used to reconstruct the elastic demand model into a fixed demand model, an approximate solution is given, the three factors of path time and reliability are integrated, dynamic travel cost is generalized, and users are simulated to make a path with the criterion of the minimum estimated dynamic cost. The stochastic user equilibrium model is established under the elastic demand condition, and the continuous average algorithm (MSA) for solving the model is given. The convergence of the fluctuation of road section travel time with the change of road length under the elastic demand condition is studied [11], the travel process of vehicles in the road network is simulated by using MITSIM simulation, and the research results show that the road section travel time converges with the increase of road length under the elastic demand condition.

The characteristics and stability conditions of the dynamic evolution of traffic flow are analyzed, and the Logitbased SUE problem under elastic demand is studied, which not only models the route choice of travelers according to the SUE principle but also estimates the traffic demand by considering the influence of the expected travel time of the road section on the traffic demand, and constructs a twolayer planning model based on the Logit-SUE problem for
The problem of difficulty in solving the elastic demand traffic assignment model is designed to achieve the user equilibrium state through adaptive adjustment in the iterations by an effective algorithm. The traffic paradox under different equilibrium conditions of fixed and elastic traffic demand, including user equilibrium and stochastic user equilibrium, is studied comprehensively, and the average travel [12] cost between OD pairs is chosen as the main index to discern whether there is a traffic paradox. Finally, the effects of travelers' perceptual errors and travel cost sensitivity on the occurrence of elastic transportation systems are analyzed.

\section{Algorithm Design}

Tourists are the main body of tourism and the basic element of the three major elements of tourism. Without tourists, natural tourism cannot be realized. Tourists, literally interpreted, are tourists; that is, people engaged in tourism activities. The topological method of super transportation network based on the urban road network, urban bus line network, and rail line network is used to combine the three networks into one through virtual interchange nodes and virtual interchange sections to form an urban super transportation network. A multinomial random path selection algorithm based on the super transportation network is proposed and its parameters are calibrated using the great likelihood method. In the upper model, the objective is to maximize the social benefits of the transportation system considering the construction cost of the hub [13]. In the lower layer model, the user balance model of the super network is used to realize the nonset count selection of passenger travel paths and traffic assignment distribution, and a forbidden search algorithm is proposed to solve the interchange hub layout optimization model. Traffic flow distribution is an important part of traffic network design and traffic management, which is to distribute the predicted traffic volume to each road section according to certain criteria based on the road network structure, impedance, and other conditions and, further, find the traffic volume and impedance of each road section and use it to evaluate the traffic network condition. According to whether the traffic demand varies with the degree of network congestion, it can be divided into fixed demand and elastic demand traffic allocation models. Elastic demand means that when congestion increases between an OD pair in the road network, some travelers will change their travel time or cancel their trips, and the traffic demand between ODs will decrease. Elastic demand traffic assignment is an extension of the study of fixed demand, and many research results have been obtained in recent years. The most basic one is the elastic demand user equilibrium (EDUE) model, which can be solved to obtain the roadway flow and OD demand satisfying the Wardrop user equilibrium principle, and the OD demand must also satisfy the defined demand function.

The two-level planning model constructed in the literature belongs to the nonlinear planning problem, which is the key problem in constructing the flexible transportation algorithm, and also a multiobjective optimization problem. Generally speaking, there is no unique optimal solution for 
the multiobjective problem, and there is a game between multiple objectives, which cannot reach the optimum at the same time, so this paper transforms the multiobjective problem into a single-objective problem by weighting coefficients. The solution algorithms of two-level nonlinear programming mainly include descent algorithm, artificial bee colony algorithm, and genetic algorithm. The descent algorithm is often easy to get the local optimal solution, and the intelligent algorithm is more suitable for the solution of the two-level programming model [14]. Considering the characteristics of discrete decision variables in the upperlevel model, this paper designs the artificial bee colony algorithm to solve the two-level planning model for one-way traffic organization optimization. The swarm algorithm is an intelligent algorithm that imitates the behavior of a bee colony to find a good food source. By comparing the advantages and disadvantages of the solution sets generated randomly and with objectives, the global optimal solution is finally obtained by an individual local search of the hiring bees, observation bees, and detection bees, and the convergence speed is faster. Compared with the genetic algorithm, the artificial bee colony algorithm is designed with neighborhood search and thus has a faster convergence speed and better local optimization search capability.

Using the real number coding method, firstly, the branch road sections in the one-way traffic organization area are numbered, $\mathrm{B}$ is the number of branch road sections, each section corresponds to a coding position, the coding value of the corresponding position is determined according to the direction of travel of the section, and the coding value range is $\{0,1,-1\}$. All branch road sections in the network are sequentially encoded, forming a vector of length B. Figure 1 shows the encoding diagram of the solution [15].

In the swarm algorithm, a new solution is generated by a neighborhood search operation based on the current solution. In this paper, we propose a probabilistic reverse-orderand-swap neighborhood search strategy, which generates two sets of random numbers in the range of 1 to $B$ without overlap, extracts two sequences based on the range of random numbers inside each solution, reverses the sequences separately, and then swaps the positions of the two sequences in the code. See Figure 1.

For any solution $y$ generated by the domain search, it is brought into the lower layer to find out the traffic flow and load degree of the corresponding road section in equilibrium by the SUE model, and then, the objective function value of the upper layer problem is calculated. The upper layer problem is a minimization problem, and the fitness function is designed according to the objective function $Z(y)$, for any given coding sequence. corresponding to the solution $y$, the fitness value is defined as follows.

$$
\text { Fitness }(y)=Z_{\max }-Z(y), \quad Z(y)>Z_{\max } .
$$

The common food source selection strategies used in swarming algorithms are roulette selection and tournament selection methods.

The fitness value calculates the sharing probability of the food source under the selected strategy to ensure that the good food source is passed on with maximum probability. In this paper, the roulette selection method is used for the selection of a good food source, i.e., the optimal feasible solution, through which the strategy ensures that good individuals are passed on to the next generation with maximum probability without being lost in the evolutionary process and accelerates the convergence of the algorithm [16].

The basic steps for the solution of the artificial bee colony algorithm designed in this paper are given as follows.

\section{Step 1: Initial solution generation}

Randomly generate $n$ initial solutions $\{Y i\}(i=1,2,3$, $\ldots, n)$; let iter $=0$ and neighborhood search count $L i=0(i=1,2,3, \ldots, n)$; set the upper limit of the number of iterations of neighborhood search Limit Num and the maximum number of iterations of the algorithm Max Iter Num.

Step 2: Hire bee stage

A neighborhood search is performed for each solution $Y i$, in turn, to generate a new solution, $Y i$, comparing the magnitude of the fitness function value of each solution in the original solution set $X$ with that of the new solution generated by the corresponding search.

Step 3: Observation bee stage

The roulette selection method is used to randomly select one solution ${ }_{\mathrm{Yi}}$ for neighborhood search and generate a new solution Fitness $(X)>\operatorname{Fitness}(X)$, then replace $Y$ with $X$, and update the solution in $X$ so that $L i=0$; conversely, let $L i=L i+1$; repeat the process $n$ times.

Step 4: Scouting bee stage

For any solution $Y i$, if the number of neighborhood searches $L i=$ Limit Num, the old solution is discarded and a new solution $Y i$ is randomly generated instead.

\subsection{Resilient Equilibrium Model Solving Based on an Elastic} Traffic Network Algorithm. The algorithms currently used to solve the SUE model based on discrete choice include Dial algorithm, iterative weighting (MSA), and Clark approximation. The Clark approximation is only applicable to the solution of the Probit model, and the Dial algorithm is only applicable to the solution of the Logit model. Sheffi and Powell first proposed the Method of Successive Averages (MSAs) in 1982 for solving the mathematical programming model of the SUE equivalent model [17].

Successive averaging is a cyclic allocation method between the balanced allocation method and the incremental allocation method, also known as the successive averaging method or the quadratic weighted averaging method. The basic idea of the algorithm is to gradually approach the equilibrium solution by adjusting the loading flow rate of each road segment. Each iteration is predetermined in steps, and the traffic flow is allocated by a random loading method (such as Dial algorithm), and the cycle is continued until the difference of the traffic volume allocated to each road section 


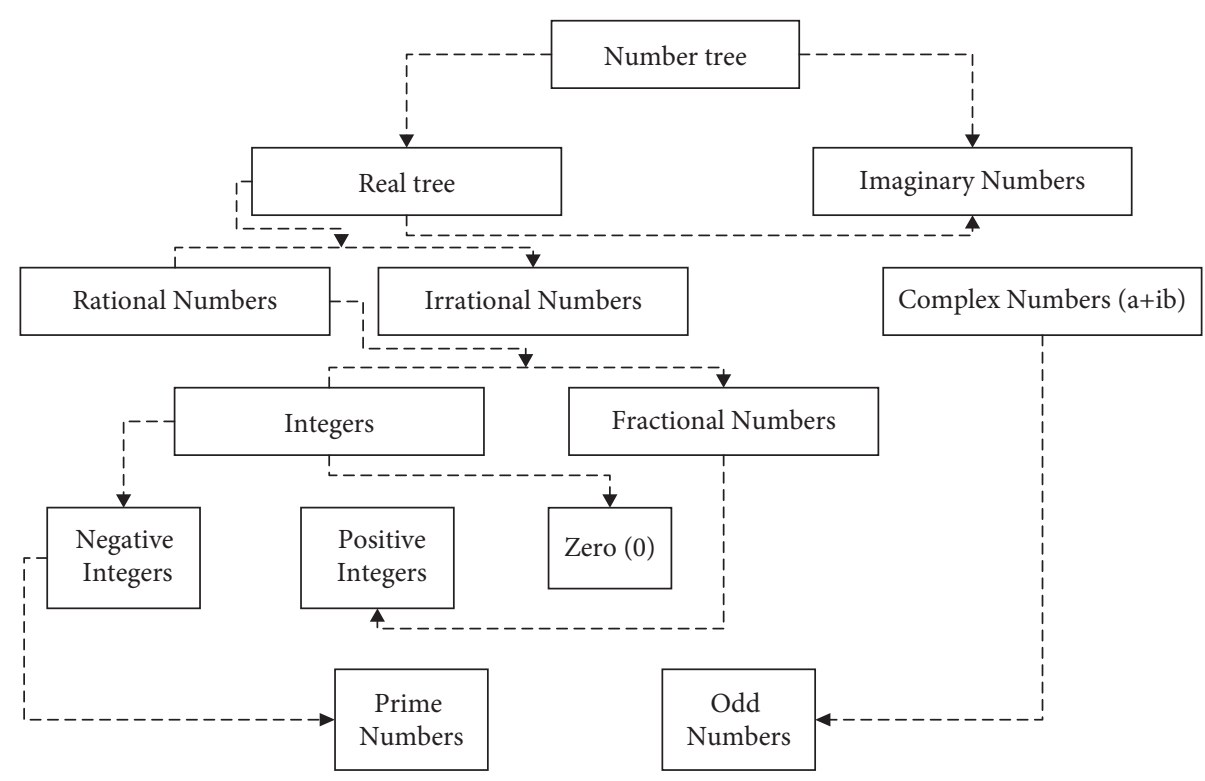

Figure 1: Principles of number sequence conversion.

in two iterations is less than the given error limit; then, the equilibrium solution is obtained. In response to the shortcomings of single path assignments, scholars have proposed random loading assignment methods, which are mainly divided into two types.

Decoding is a process of using a specific method to restore the digital to the content it represents or to convert electrical pulse signals, optical signals, radio waves, etc. into the information, data, etc. it represents. Decoding is the process by which the receiver restores the received symbols or codes to information, which corresponds to the encoding process.

Logit Method and Probit Method. To solve the Logit-SUE model proposed in this paper, the solution algorithm of the Logit model is highlighted here.

The Dial algorithm enables efficient implementation of the Logit model on the network in the following steps [18].

Step 1: Determine the effective paths and road sections. Calculate the minimum impedance from the starting point $r$ to all nodes, denoted as $r(i)$; calculate the minimum impedance from all nodes to the end point $s$, denoted as $s(i)$; denote $\mathrm{O}_{i}$ as the set of section endpoints with the starting point $I$; define $D_{i}$ as the set of section start points with the endpoint $i$.

$$
L(i, j)=\frac{\delta y}{\delta x}, \sum_{i=i}^{n} X_{j}^{2} .
$$

Step 2: Starting from the starting point $r, r(i)$ increases in order, and calculate the weight of each node leaving its data section according to the above formula:

$$
w(i, j)=\sum_{i=1}^{n}\left(j_{i}-\bar{X}\right)^{2}
$$

The weight calculation stops when the end point $s$, i.e., $i=s$, is reached.

Step 3: Starting from the end point $s$, calculate the traffic volume of the road section in the ascending order of $s(j)$.

The computation stops when the starting point $r$, i.e., $j=r$, is reached.

It can be shown that the results obtained by the Dial algorithm are identical to the assignment results of the Logit model; i.e., the Dial algorithm and the Logit model are equivalent.

MSA algorithm is widely used, is simple to calculate, and is the closest to the equilibrium distribution method of a flow distribution method which is the classical algorithm for solving the SUE model; the solution steps can be summarized as follows.

Step 1. Initialization: Let $0, X a=A \Omega B$, get the initial impedance of each road section, and accordingly, the traffic flow between OD pairs is loaded randomly (this paper uses the Dial algorithm mentioned above) to get the initial road section flow $0 \mathrm{ax}$, so that the number of iterations $n=0$.

Step 2. Update the impedance of each road section, according to the current traffic volume of each road segment $X a$.

Step 3. Search for the direction of descent: According to the new impedance obtained from Step 2, the Dial algorithm is invoked to randomly load the traffic flow between ODs to obtain the additional flow $\mathrm{Fa}$ of the road section.

Step 4. Update road traffic.

Step 5. Convergence conditions: If the formula meets the above requirements, 


$$
w(i, j)=\sum_{i=1}^{n} X_{i} Y_{i}
$$

\subsection{Flexible Traffic Network Allocation Model}

3.2.1. Elastic Demand Model Construction. Elastic demand refers to the variation of OD traffic demand with the degree of network congestion. Its formula is as follows:

$$
\min Z_{\mathrm{EDUE}}(x, Q)=\sum_{i=1}^{n} X_{i}^{2} \int \sum_{i=1}^{n} X_{i} Y_{i}-\sum_{i=1}^{n} X_{i}^{2} \int \sum_{i=1}^{n} X_{i} Y_{i} \text {. }
$$

Usually, $D(r s)$ is a monotonically decreasing (at least not increasing) function of the travel resistance between OD pairs $(r, s)$. In addition, the demand function is still online because the population size and car ownership of each node are limited functions. In summary, the EDUE model can be described by the following mathematical planning model:

$$
\min Z_{\mathrm{EDUE}}(x, Q)=\sum_{i=1}^{n} X_{i}^{2} \int \sum_{i=1}^{n} X_{i} Y_{i}-\sum_{i=1}^{n} X_{i}^{2} \int \sum_{i=1}^{n} X_{i} Y_{i} .
$$

Based on the above description, the change in the demand function to the excess demand function of the public in terms of travel can be derived, and the image of the function is as follows. Figure 2 shows changes in demand function to excess demand function.

In the elastic demand (EDUE) model, the traffic demand between OD pairs will be adjusted according to the change of travel impedance, and the process of network traffic equilibrium under the elastic demand condition is based on the degree of network congestion. Figure 3 shows the transformation diagram of the excess demand network. Supply and demand balance to determine the right demand for each OD pair.

\subsubsection{Definition and Calculation Model of Road Network} Resilience Index. To quantitatively analyze the resilience of the road network to disasters, the concept of the "road network resilience index" is proposed to characterize the ability of the road network to dissipate itself when it is partially damaged. When a path network is partially damaged, the efficiency of the network decreases or fails partially, a high resilience index of the network or nodes indicates that the network can absorb and dissipate the consequences better, and the network is resilient. The topological resilience of each node constitutes the topological resilience of the whole road network [19].

When the efficiency of a node of a network decreases or fails due to a disaster, the ability of its surrounding network to absorb and dissipate the effects of its failure is the node resilience index. The absorption capacity of all nodes constitutes the absorption capacity of the whole network. The road network resilience index is described from two aspects: (i) as a complex network, the network itself has a large impact on the resilience, so it is necessary to consider the topological characteristics of the road network; (ii) the traffic

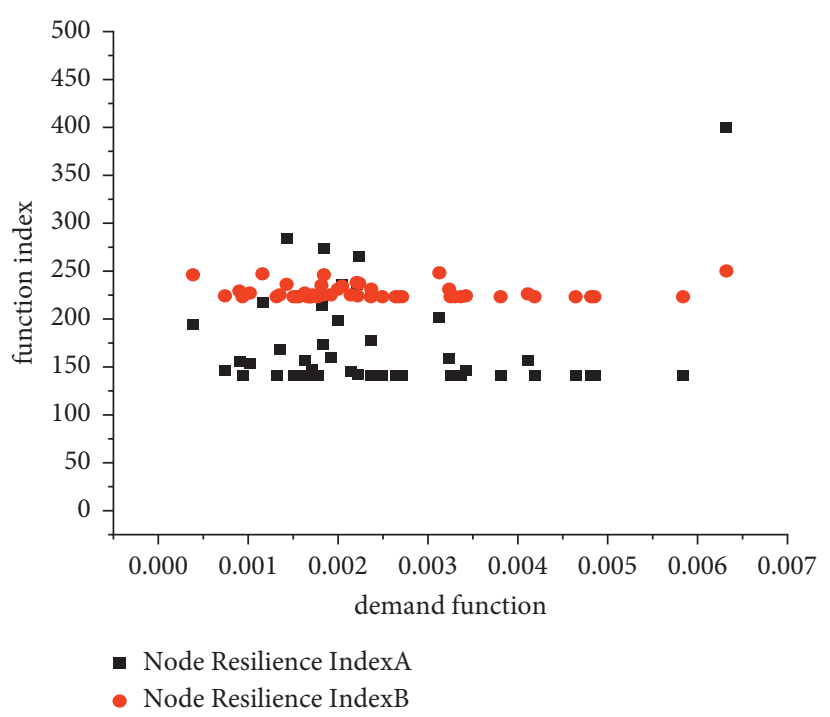

Figure 2: Changes in demand function to excess demand function.

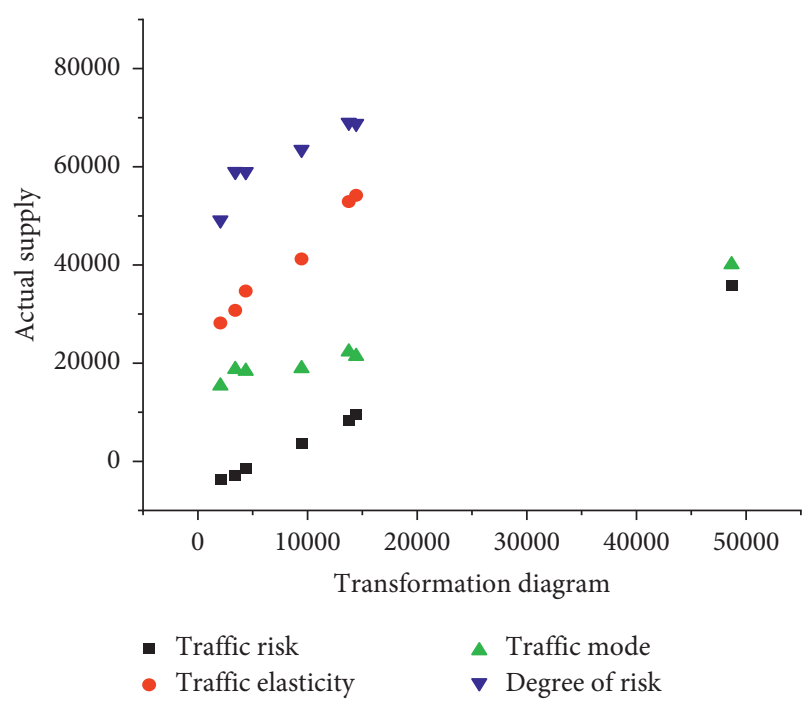

Figure 3: Transformation diagram of excess demand network.

characteristics of the road reflect the number and spatial and temporal distribution of traffic on the road, which is an important constraint on the resilience of the road network. The traffic characteristics on the road are often neglected in the previous studies of road network resilience [20]. See Figure 4.

Figure 4 displays the topology of the road network which is the "precondition" of the road network, which affects the resilience of the road network nodes to a certain extent. The topological methods commonly used to transform the topology of the actual road network are the principal method and the pairwise method. The topology of the path network is analyzed by using complex network theory, and the topological characteristics of the nodes are evaluated by using three indicators: node degree, intermediate degree, and near intermediate degree. The node degree is defined as the number of connections connected to the node, the intermediary intermediate degree reflects the importance of the 


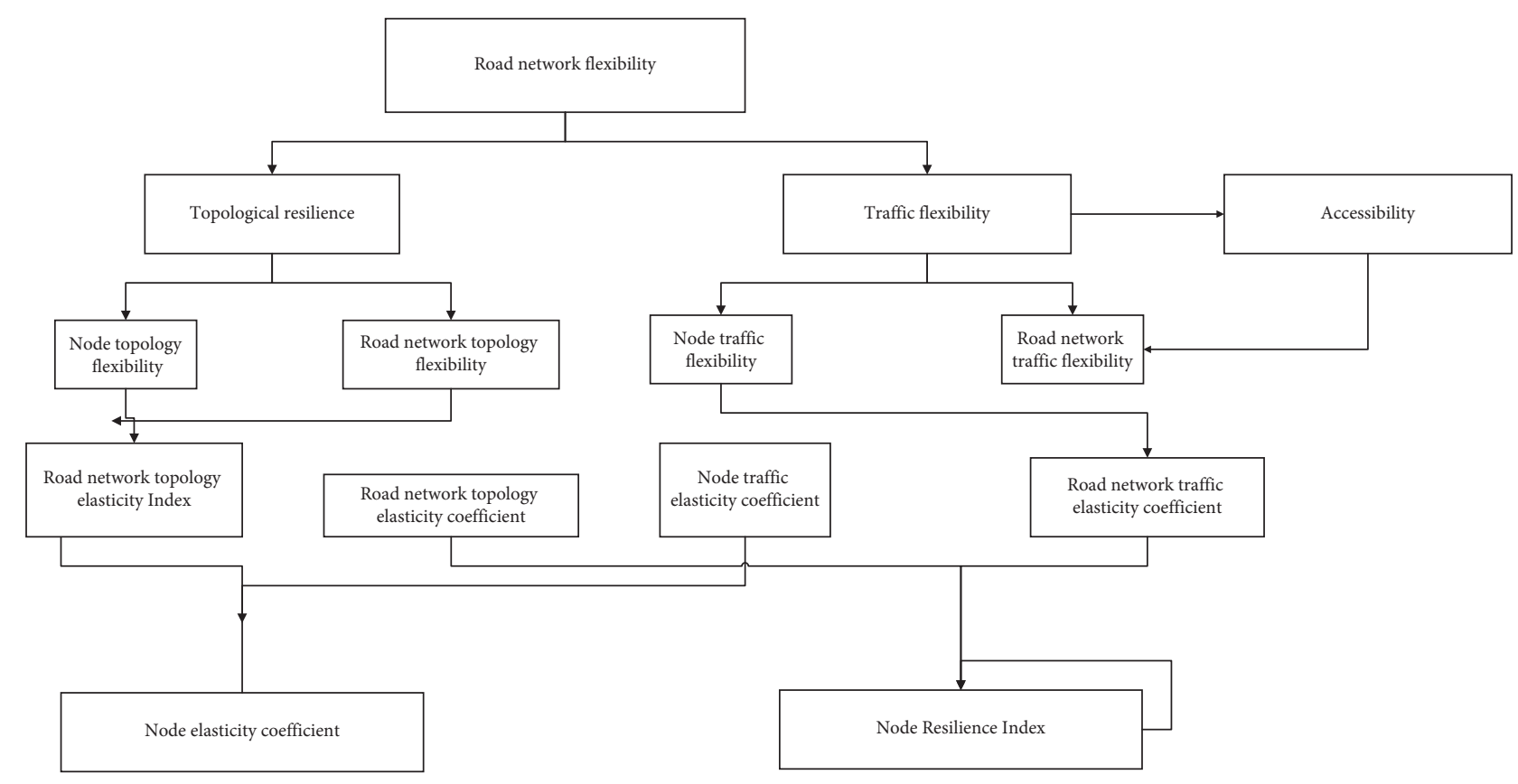

FIGURE 4: Conceptual components of the road network resilience index.

node in the network path selection, and the node with the highest degree of intermediary intermediate degree is the node with the highest number of shortest paths traversed in the network. Proximity centrality is the average shortest path between any point in the network and other nodes, which reflects the proximity of a node to other points on the network.

\section{Model Solving Algorithm}

Sensitivity analysis is a very important learning content in traffic network planning, especially in linear planning, but there are relatively few sensitivity studies applied to traffic network design, first of all, because of the complexity of traffic networks, and in many cases, it is extremely difficult to express them as nonlinear planning and let linear planning be alone. In addition, the uniqueness of the equilibrium path flow solution is not guaranteed, so it becomes very difficult to analyze the sensitivity of traffic equilibrium [21, 22]. However, it is a very effective research method in the descent method, and it is still quite effective for some specific equilibrium flow distribution models. Therefore, in recent years, more and more scholars have explored the sensitivity of traffic network equilibrium.

The nonnegative parameter 0 in the SUE model takes the value of 1 and the expansion cost function, which is the discount factor of the traffic manager's cost discounted to the expansion year during the expansion planning period. Here, we assume that the demand function is a constant 30 , and the correlation coefficients for each road segment are shown in Table 1. The two-level planning problem is solved using the sensitivity analysis algorithm as developed in this paper. See Table 1.

Analysis of the Results. We know from Table 1 the actual situation; the equilibrium traffic volume of Sections 1, 4, and
TABLE 1: Parameters related to each road section.

\begin{tabular}{lccccc}
\hline Road network & 1 & 2 & 3 & 4 & 5 \\
\hline$t_{a}$ & 5 & 6 & 9 & 56 & 5 \\
$c_{a}$ & 35 & 54 & 77 & 87 & 44 \\
$y_{a}$ & 6 & 7 & 3 & 66 & 68 \\
$K_{a}$ & 8 & 5 & 77 & 78 & 9 \\
\hline
\end{tabular}

5 is not going to change; that is, its equilibrium traffic volume and the derivative of the change in the capacity of the section are 0 . Therefore, the circulation capacity of the model will change after the expansion of only Sections 2 and 3, which is consistent with the results obtained from the calculation of Table 2, so the algorithm is reasonable and effective. See Table 2.

4.1. Road Network Model and Parameter Setting. In this paper, a district is selected for the case study, and the arterial and branch roads in the region are abstracted. The road network topology is shown in Figure 5 with 34 nodes and 56 road sections. Among them, nodes 1, 2, 3, and 4 are the starting points. The dashed lines in the figure indicate the virtual road sections added between each OD pair. See Figure 5.

Among them, because the complexity of travel of the city's residents is much more than this, so this paper constructs more complex traffic network routes, with the support of algorithms, because the residents' travel needs are complex and diverse with multiplication. Figure 6 displays the road network topology index, so there are more risky perturbations, and more travel routes need to be constructed to have true simulation. The following is the distribution of the road network with real simulation. See Figure 6. 
TABle 2: Traffic network optimization diagram.

\begin{tabular}{lccccc}
\hline Road section & 1 & 2 & 3 & 4 & \\
\hline Section traffic volume & 54 & 45 & 23 & 71 & 43 \\
Circulation capacity after expansion & 36 & 61 & 26 & 32 & 35 \\
Elastic demand coefficient & 44 & 29 & 37 & 23 \\
\hline
\end{tabular}

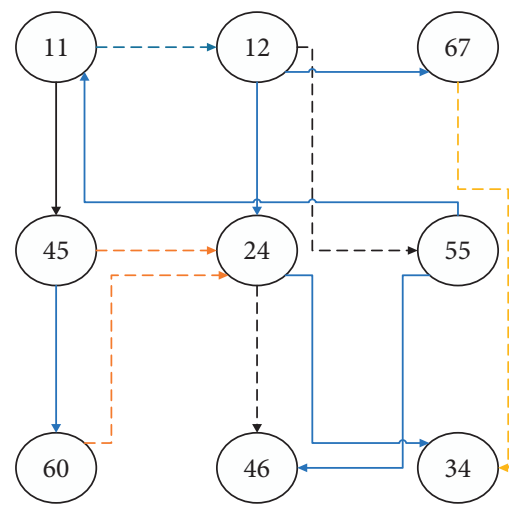

Figure 5: Road traffic network.
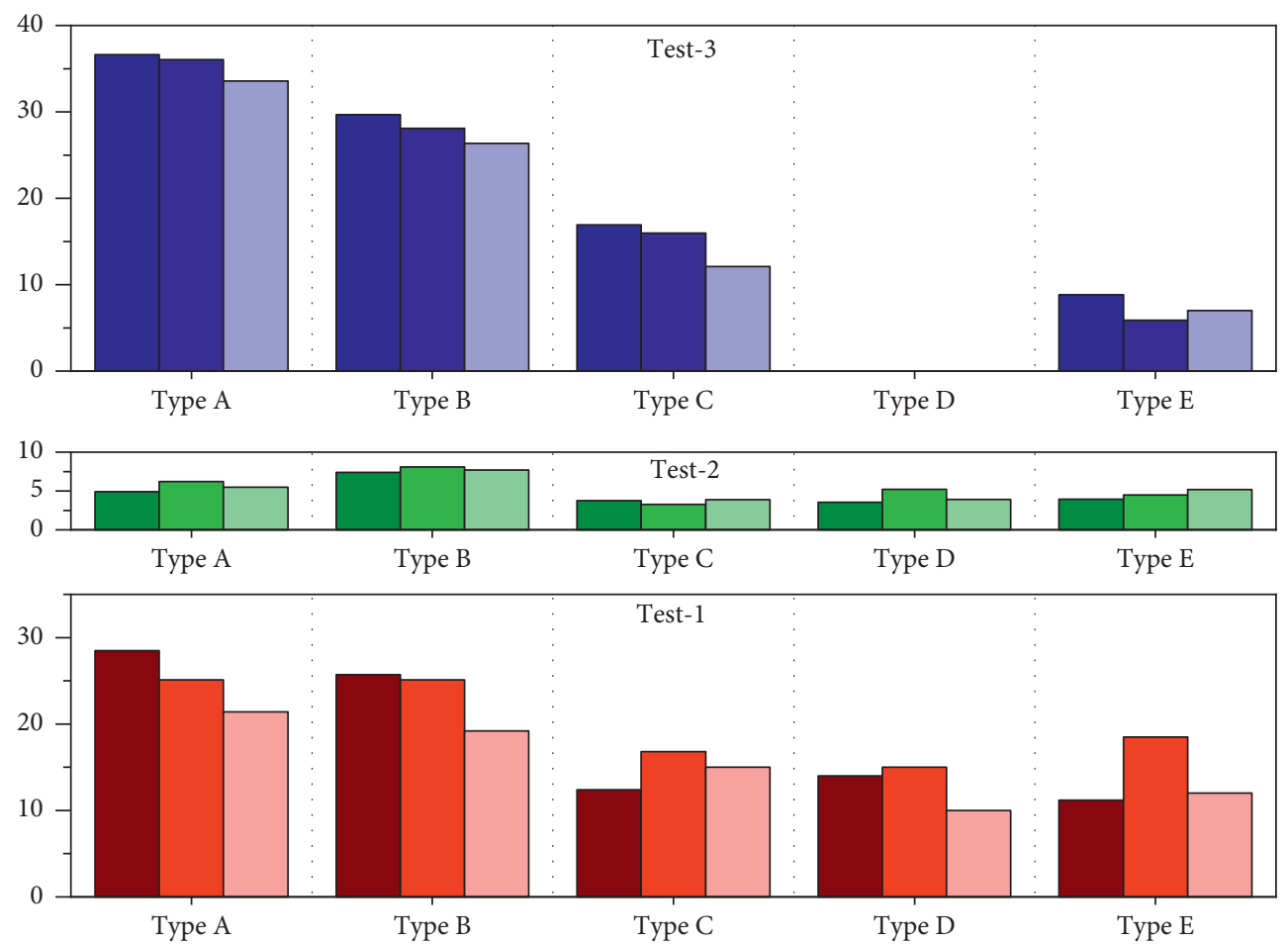

FIGURE 6: Road network topology index.

DCS subsystem is the core of the CBTC system to realize information interaction between subsystems, mainly transmitting control commands, train status, and other key information, ensuring bidirectional, safe, and reliable transmission of data, and playing an important role in the whole train control system. The following figure is the communication diagram of CBTC system subsystems; in the DCS system, there is mainly two-way information interaction between the following subsystems: ZC and VOBC, ZC and $\mathrm{Cl}, \mathrm{ZC}$ and ATS, ZC and DSU, ATS and VOBC, ATS and $\mathrm{Cl}$, ATS and DSU, and VOBC and $\mathrm{Cl}$. VOBC communicates with ground equipment through a wireless access network, and the rest of the subsystems are based on the backbone network for data transmission. See Figure 7.

By comparing various transportation system operation diagrams and actual operation diagrams, it can be found that certain riskiness can cause changes in train operation status; i.e., Figure 7 shows that there are differences in the 


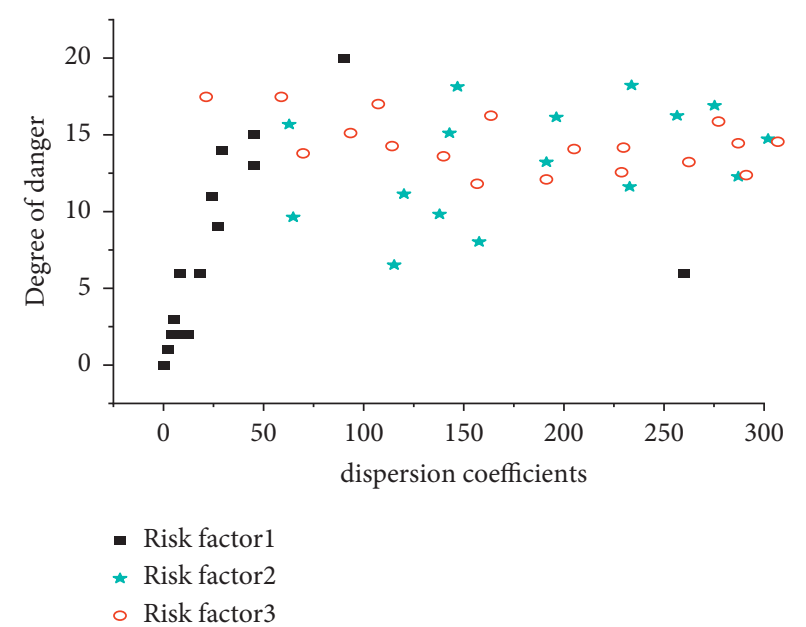

FIGURE 7: RMSE with different dispersion coefficients.

displacement of the same train traveling in the zone at the same moment before and after the system failure; plus, the driving mode of the train is different before and after the system failure, so there are also differences in the traveling speed of the same train at the same moment. Considering that the stopping time of the same train at the same station before and after the system failure may overlap, the abovementioned displacement difference and the speed difference are zero; therefore, while considering the displacement and speed difference as indicators for evaluating the change of train operation performance, the train late time is also used as a measurement indicator.

4.2. Analysis of Experimental Results. This experiment uses the same traffic network as the numerical experiment above and gives the algorithm used to calculate and assign the roadway flow when the dispersion factor takes different values.

Figure 8 shows that when suboptimal congestion charging uses different lower-level models to characterize traveler behavior, the resulting roadway charging schemes are different, which indicates that when developing congestion charging policies, it is necessary to use more. See Figure 8.

Add the traffic distribution model that conforms to the traffic network condition of the city understudy, or consider the toll results obtained from multiple models to reasonably determine the final road section toll scheme. When the dispersion coefficient increases, the lower level of the kind of multiuser multicriteria stochastic user balance model of the path section toll value is more than a multiple of the model, which indicates that when the traveler travels a greater degree of dispersion, the amount of road section congestion toll is higher than in the model. The distribution results are similar to the fact that as the dispersion coefficient increases, there is an overall decreasing trend in the traffic of road sections and an overall increasing trend in the traffic of road sections. This is because a larger dispersion coefficient indicates that the travelers are more familiar with the path network, their travel choice is closer to the deterministic

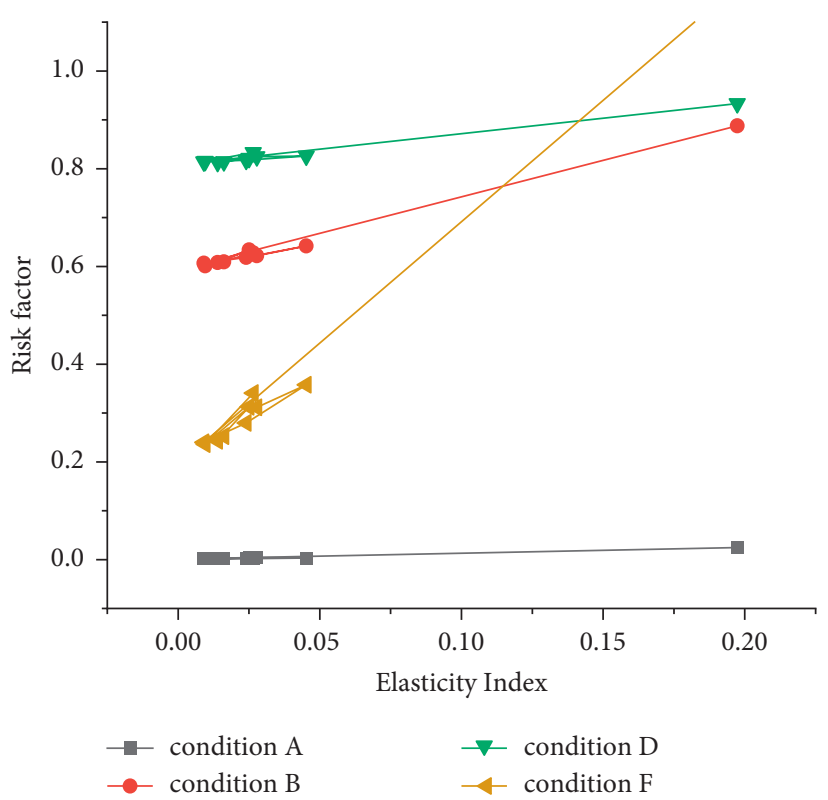

FIgURe 8: Comparison of trends in risk factors.

choice, the first term of the objective function dominates, and the travelers' travel is close to the result of user equilibrium. As the dispersion coefficient increases, the difference between the model and model section assignment results becomes smaller and smaller, which also reflects the fact that no increase leads to travelers' trips closer to deterministic choices. Regarding the complex transportation network $G$ as a system containing $n$ nodes and their interactions, there is a virtual field around each node, and any node in the network will be subject to the joint action of other nodes; thus, in the entire network, a data field is determined topologically, which is called network road resilience.

Similarly, regardless of the dispersion coefficients, the model assignment results are smaller for roadway segments and larger for roadway segments. This is because the assumption ignores the correlation between paths, and thus, the traffic assignment results for overlapping paths and overlapping path sections will be larger, while the overlapping relationship between paths is considered to adjust the negative utility of travel by paths, and the paths with a high degree of overlap are adjusted more and vice versa to a lesser extent. Thus, the model can overcome the characteristics of traditional models to some extent.

The above are the results of the resilience assessment of the system under two scenarios with riskiness, and the comparison reveals that the absorption capacity of the system resilience is comparable under both riskiness scenarios, indicating that the system has similar robustness in the face of both risks, and the adaptive capacity of the system is weaker under the first riskiness scenario, indicating that the first attack has a greater impact on the system; in addition, the relaxation time is greater than the initial system recovery time, indicating that the initial system recovery measures are effective and that scenario one is faster in human intervention to repair the system after a failure occurs, and therefore, the recovery is faster. 


\section{Conclusion}

The urban rail transit system is closely related to the people's livelihood and is one of the key infrastructures to promote the efficient operation of the city and rapid economic development. In this paper, we analyze the serious information security situation faced by the transportation system and clarify that the research and application of continuous risk perturbation assessment methods are extremely urgent and important for the train control system. At present, there are relatively few studies on continuous risk perturbation of urban railways at home and abroad, and most of them only stay at the concept and framework level; although there are a lot of information security risk assessment methods of traditional IT systems and industrial control systems to draw on, given the unique properties of urban transportation systems, the relevant methods cannot be fully applied, so this paper focuses on the study of resilient transportation under continuous risk perturbation. Therefore, this paper focuses on the risk assessment method for resilient traffic under continuous risk perturbation. The resilient transportation method and its application play an important role in improving the information security capability of urban rail transportation.

In this paper, based on the detailed analysis of the structure and function of a typical urban transportation system one-by-one communication-based train operation control (CBTC) system, the concept of vulnerability index is proposed to assess the vulnerability of each device, scenario, and the whole system in CBTC; secondly, concerning the relationship between all devices in the resilient transportation system from the perspective of network structure, a complex network model is constructed, and a comprehensive information domain and physical domain CBTC system information security risk assessment method is proposed; finally, based on the resilience theory, the CBTC system information security resilience assessment model is proposed as an indicator, and there are model construction and algorithm implementation for quantitative analysis of column control system information security.

The attack tree model and the construction of the complex network model proposed in this paper are realized artificially. As the scale of the system increases and the connection between devices becomes more and more complex, the workload of model construction will increase greatly, and how to design and develop automatic model generation tools can be a subsequent research topic. The attack scenarios simulated in this paper are limited, and the attack process is determined by humans; how to combine the characteristics of the column control system to establish a perfect attack library and simulate the automated attack process is of great significance for the subsequent research related to the information security of the column control system. In the future, the algorithm of the article will mainly focus on improving the timeliness of the algorithm, so that congested roads can be diverted, to solve the phenomenon of traffic congestion.

\section{Data Availability}

The data used to support the findings of this study are included within the article.

\section{Conflicts of Interest}

The authors declare that there are no conflicts of interest.

\section{Acknowledgments}

This paper was not funded by any organization. The authors would like to acknowledge the Scientific Research Project of Colleges and Universities in Inner Mongolia Autonomous Region (NJZY21160).

\section{References}

[1] T. C. Sharkey, S. G. Nurre Pinkley, D. A. Eisenberg, and D. L. Alderson, "In search of network resilience: an optimization-based view," Networks, vol. 77, no. 2, pp. 225-254, 2021.

[2] D. Ivanov, B. Sokolov, and A. Dolgui, "The Ripple effect in supply chains: trade-off "efficiency-flexibility-resilience" in disruption management," International Journal of Production Research, vol. 52, no. 7, pp. 2154-2172, 2014.

[3] L. Zhang, G. Zeng, D. Li, H.-J. Huang, H. E. Stanley, and S. Havlin, "Scale-free resilience of real traffic jams," Proceedings of the National Academy of Sciences, vol. 116, no. 18, pp. 8673-8678, 2019.

[4] A. Javed and Y. Kohda, "Sustainable work opportunities for drivers' well-being: a case of careem as transportation network company," in Proceedings of the International Conference on Applied Human Factors and Ergonomics, pp. 70-77, Springer, New York, NY, USA, July 2021.

[5] M. Diao, H. Kong, and J. Zhao, "Impacts of transportation network companies on urban mobility," Nature Sustainability, vol. 4, no. 6, pp. 494-500, 2021.

[6] I. Dakic, K. Yang, M. Menendez, and J. Y. J. Chow, "On the design of an optimal flexible bus dispatching system with modular bus units: using the three-dimensional macroscopic fundamental diagram," Transportation Research Part B: Methodological, vol. 148, pp. 38-59, 2021.

[7] C. Li and B. Palanisamy, "Reversible spatio-temporal perturbation for protecting location privacy," Computer Communications, vol. 135, pp. 16-27, 2019.

[8] S. Mohammed, J. Fiaidhi, and M. Tang, "Towards using micro-services for transportation management systems," LISS 2020: Proceedings of the 10th International Conference on Logistics, Informatics and Service Sciences, Springer, Singapore, pp. 107-117, 2021.

[9] F. Goodarzian, D. Shishebori, H. Nasseri, and F. Dadvar, “A bi-objective production-distribution problem in a supply chain network under grey flexible conditions," RAIRO-Operations Research, vol. 55, pp. S1287-S1316, 2021.

[10] M. Nogal and D. Honfi, "Assessment of road traffic resilience assuming stochastic user behaviour," Reliability Engineering \& System Safety, vol. 185, pp. 72-83, 2019.

[11] K. Govindan, M. Fattahi, and E. Keyvanshokooh, "Supply chain network design under uncertainty: a comprehensive review and future research directions," European Journal of Operational Research, vol. 263, no. 1, pp. 108-141, 2017. 
[12] I. M. Cavalcante, E. M. Frazzon, F. A. Forcellini, and D. Ivanov, "A supervised machine learning approach to datadriven simulation of resilient supplier selection in digital manufacturing," International Journal of Information Management, vol. 49, pp. 86-97, 2019.

[13] N. U. Ibne Hossain, M. Nagahi, R. Jaradat, C. Shah, R. Buchanan, and M. Hamilton, "Modeling and assessing cyber resilience of smart grid using Bayesian network-based approach: a system of systems problem," Journal of Computational Design and Engineering, vol. 7, no. 3, pp. 352-366, 2020.

[14] G. Como, "On resilient control of dynamical flow networks," Annual Reviews in Control, vol. 43, pp. 80-90, 2017.

[15] F. O. Olowononi, D. B. Rawat, and C. Liu, "Resilient machine learning for networked cyber physical systems: a survey for machine learning security to securing machine learning for CPS," IEEE Communications Surveys \& Tutorials, vol. 23, no. 1, pp. 524-552, 2020.

[16] D. Xue and S. Hirche, "Finite-time distributed topology design for optimal network resilience," IET Control Theory \& Applications, vol. 13, no. 17, pp. 2792-2799, 2019.

[17] Y.-P. Fang and E. Zio, "An adaptive robust framework for the optimization of the resilience of interdependent infrastructures under natural hazards," European Journal of Operational Research, vol. 276, no. 3, pp. 1119-1136, 2019.

[18] T. N. Turan, J. H. Voeks, M. I. Chimowitz et al., "Rationale, design, and implementation of intensive risk factor treatment in the CREST2 trial," Stroke, vol. 51, no. 10, pp. 2960-2971, 2020.

[19] J. Lighter, M. Phillips, S. Hochman et al., "Obesity in patients younger than 60 years is a risk factor for COVID-19 hospital admission," Clinical Infectious Diseases, vol. 71, no. 15, pp. 896-897, 2020.

[20] D. Ivanov and B. Sokolov, "Simultaneous structural-operational control of supply chain dynamics and resilience," Annals of Operations Research, vol. 283, no. 1, pp. 1191-1210, 2019.

[21] Z. Lin, F. Long, Y. Yang, X. Chen, L. Xu, and M. Yang, "Serum ferritin as an independent risk factor for severity in COVID19 patients," Journal of Infection, vol. 81, no. 4, pp. 647-679, 2020.

[22] D. Hutchison and J. P. G. Sterbenz, "Architecture and design for resilient networked systems," Computer Communications, vol. 131, pp. 13-21, 2018. 